\title{
Inhibition of tumor growth by a newly-identified activator for epidermal fatty acid binding protein
}

\author{
Enyu Rao ${ }^{1}$, Puja Singh ${ }^{1}$, Xiuhong Zhai ${ }^{1}$, Yan Li $^{1}$, Ganqian Zhu ${ }^{1}$, Yuwen Zhang ${ }^{1}$, \\ Jiaqing Hao ${ }^{1}$, Young-In Chi ${ }^{1}$, Rhoderick E. Brown ${ }^{1}$, Margot P. Cleary ${ }^{1}$ and Bing $\mathbf{L i}^{1}$ \\ ${ }^{1}$ The Hormel Institute, University of Minnesota, Austin, MN, USA \\ Correspondence to: Bing Li, email: bli@hi.umn.edu \\ Keywords: E-FABP, tumor associated macrophages, interferon $\beta$, tumor treatment \\ Received: November 20, $2015 \quad$ Accepted: February 04, $2015 \quad$ Published: March 08, 2015
}

This is an open-access article distributed under the terms of the Creative Commons Attribution License, which permits unrestricted use, distribution, and reproduction in any medium, provided the original author and source are credited.

\section{ABSTRACT}

Our previous studies have demonstrated that expression of epidermal fatty acid binding protein (E-FABP) in tumor associated macrophages (TAMs) promotes macrophage anti-tumor activity by enhancing IFN $\beta$ responses in tumor models. Thus, E-FABP represents a new protective factor in enhancing tumor immune surveillance against tumor development. Herein, we report the compound 5-(benzylamino)-2(3-methylphenyl)-1,3-oxazole-4-carbonitrile (designated EI-05) as a novel E-FABP activator for inhibition of mammary tumor growth. EI-05 was selected from the ZINC compound library using molecular docking analysis based on the crystal structure of E-FABP. Although EI-05 is unable to bind E-FABP directly, it significantly increases E-FABP expression in macrophages during inflammation. Stimulation of macrophages with EI-05 remarkably enhances lipid droplet formation and IFN $\beta$ production, which further promotes the anti-tumor activity of macrophages. Importantly, administering EI-05 in vivo significantly inhibits mammary tumor growth in a syngeneic mouse model. Altogether, these results suggest that EI-05 may represent a promising drug candidate for anti-tumor treatment through enhancing E-FABP activity and IFN $\beta$ responses in macrophages.

\section{INTRODUCTION}

Tumor initiation and progression are complex processes which depend not only on intrinsic alterations in tumor cells, but also on the extrinsic factors from host immunity. How to enhance tumor immune surveillance remains one of the most active research areas in tumor immunology $[1,2]$. Emerging evidence indicates that energetic metabolism of immune cells profoundly impacts their functions and fates [3]. Therefore, investigating the role of distinct metabolic pathways in immune populations and uncovering how these pathways determine their immunologic consequences represent important foci for the control of tumor development.

Fatty acid binding proteins (FABPs) represent a family of small and highly homologous cytoplasmic lipid chaperones that regulate lipid transfer and responses inside cells. Accumulating data have shown that FABPs are central regulators of both metabolic and inflammatory pathways $[4,5]$. While most FABP members, such as liver FABP (L-FABP) and intestinal FABP (I-FABP), exhibit tissue-specific patterns of distribution, epidermal FABP (E-FABP) displays a more ubiquitous expression, particularly in immune cells, suggesting an essential role of E-FABP in regulating energy metabolism and functions of immune populations [6, 7]. In our studies focusing on the role of E-FABP in tumor development, we have demonstrated that E-FABP deficiency in mice accelerates mammary tumor growth through impairing IFN $\beta$ responses in tumor associated macrophages (TAMs), suggesting E-FABP as a new protective factor in host against tumor development [8]. In addition, we also have reported that E-FABP expression in mice promotes the development of experimental autoimmune encephalomyelitis (EAE) [9]. While effective anti-tumor immune responses require the expression of E-FABP, overactivity of E-FABP in immune cells may instead promote inflammatory autoimmune responses. Thus, our studies suggest that E-FABP functions as an important immune regulator in shaping immune functions in 
different disease settings, and modulating E-FABP activity may provide an attractive approach for treatment of tumor and autoimmune diseases.

In fact, the use of pharmacological agents to modify FABP function has been proposed in many studies to control inflammatory responses and metabolic syndromes, thus offering a new class of multi-indication therapeutic agents [10-13]. In our efforts to screen for small molecule compounds that may regulate E-FABP activity for potential clinical application, we identified one compound 5-(benzylamino)-2-(3-methylphenyl)-1,3-oxazole-4carbonitrile (designated EI-05) which enhances E-FABP expression in macrophages and promotes their type I IFN $\beta$ responses during inflammation. More importantly, administration of this newly-identified compound significantly inhibited mammary tumor growth in vivo.

\section{RESULTS}

\section{Screening of potential E-FABP modifiers by molecular modeling}

As identification of small molecule inhibitors of FABP has been proven to be effective in treating atherosclerosis and type II diabetes in mouse models [14], we initially intended to screen small molecules that might specifically bind to the lipid-binding pocket of E-FABP using computational docking analysis [15]. Based on the crystal structure of E-FABP (PDB ID 1B56), EI-05 (Figure 1A) was selected as a potential E-FABP partner due to the predicted binding of E-FABP/EI-05 complex shown by molecular docking modeling (Figure 1B). However, when we purified recombinant E-FABP proteins and conducted in vitro binding assays, we found that E-FABP did not bind to EI-05, despite good binding to the known inhibitor BMS309403 [16], in thermal shift assays (Figure 1C). As EI-05 exhibited a relatively low excitation signal at $270 \mathrm{~nm}$ (Figure 1D), which enabled this wavelength to be used to excite Tyr and Trp in E-FABP, we evaluated the binding of E-FABP/EI-05 by Förster resonance energy transfer based on the spectral overlap of the E-FABP Tyr/ Trp emission and EI-05 excitation signals. Step-wise addition of EI-05 to E-FABP did not affect the emission of E-FABP Tyr/Trp (Figure 1E). The strong stepwise increases in EI-05 emission signal at $394 \mathrm{~nm}$, shown in Figure 1E, were nearly the same in the absence of E-FABP (Figure 1F), consistent with no energy transfer. In contrast, positive controls performed with BMS309403 showed the expected dose-dependent increase of E-FABP Tyr/Trp emission (Figure $1 \mathrm{G}$ ). Thus, although predicted to bind E-FABP by computational modeling, our in vitro binding assays clearly indicate that EI-05 has no direct binding to E-FABP.

\section{EI-05 enhances E-FABP expression in activated macrophages}

When we activated a macrophage cell line with LPS in the presence or absence of EI-05 and other potential E-FABP partners identified by computational modeling analysis, we found that EI-05, but not other small molecules, significantly enhanced E-FABP expression in macrophages (Figure 2A). We further investigated the effect of EI-05 on E-FABP expression with primary GMCSF-induced macrophages derived from mouse bone marrow (GM-BMMs). We demonstrated that E-FABP expression in EI-05-stimulated macrophages was about 4.5 fold higher than that in control groups (Figure 2B). Consistent with these in vitro observations, when EI-05 was administered in vivo, it was able to greatly enhance E-FABP expression in macrophages of the peripheral blood as shown by western blot analysis (Figure 2C). Confocal microscopy staining further demonstrated that EI-05 treatment greatly enhanced E-FABP expression in the cytoplasm of macrophages (Figure 2D). Thus, these data suggest that EI-05 can activate macrophages through enhanced E-FABP expression in both in vitro and in vivo conditions.

\section{EI-05 promotes IFN $\beta$ production in macrophages}

As E-FABP expression in TAMs can promote IFN $\beta$ responses [8], we next analyzed whether EI-05 treatment impacts IFN $\beta$ production in macrophages. Indeed, addition of EI-05 greatly enhanced IFN $\beta$ mRNA levels in LPS-activated GM-BMMs (Figure 3A) in a dose-dependent manner. Similarly, IFN $\beta$ protein levels in the culture supernatants were also positively elevated in response to increasing concentrations of EI-05 (Figure 3B). As leaking DNA from cellular damage can induce IFN $\beta$ production [17], we analyzed the cytotoxicity of EI05 on macrophages, and demonstrated a minimal impact of EI-05 on macrophage death (Figure 3C), suggesting that a specific effect of IFN $\beta$ production was induced by EI-05. When we measured IFN $\beta$ production using E-FABP WT and KO macrophages, we found that EI-05 treatment promoted E-FABP and IFN $\beta$ production in the WT cells, but not in the E-FABP KO cells (Figure 3D, $3 \mathrm{E}$ ), indicating an E-FABP-dependent effect for EI-05induced IFN $\beta$ production in macrophages. In our previous studies, we have shown that E-FABP-promoted lipid droplet (LD) formation was positively associated with IFN $\beta$ production [8]. It is likely that EI-05 treatment may promote IFN $\beta$ production through E-FABP-promoted LD formation. To this end, we measured the impact of EI-05 on LD formation in macrophages. Confocal microscope analysis showed that EI-05 greatly upregulated LD formation in macrophages (Figure 3F). In agreement with our previous results, EI-05-enhanced LD formation and 
A

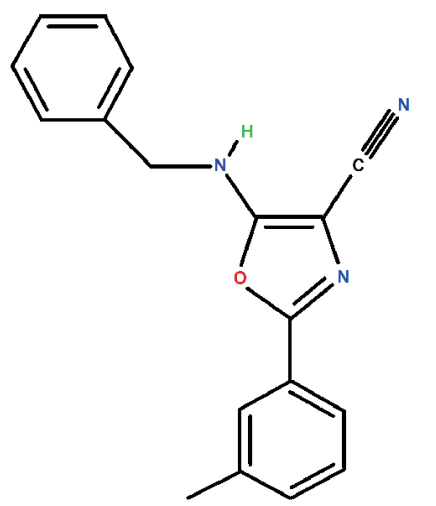

C

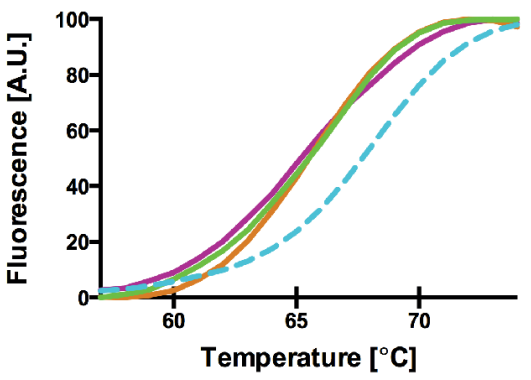

$E$

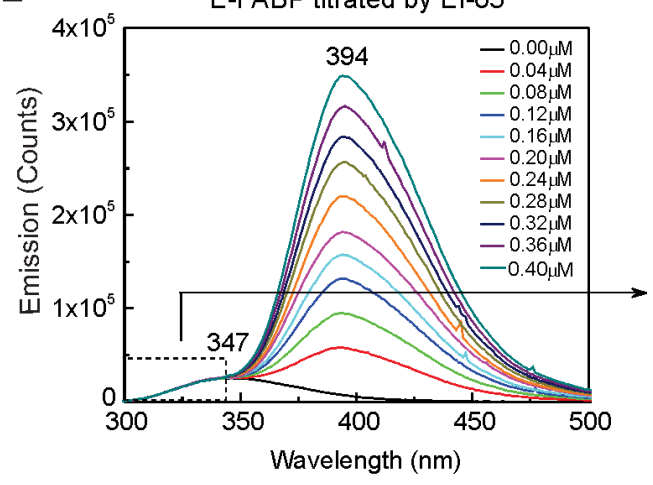

$\mathbf{F}$

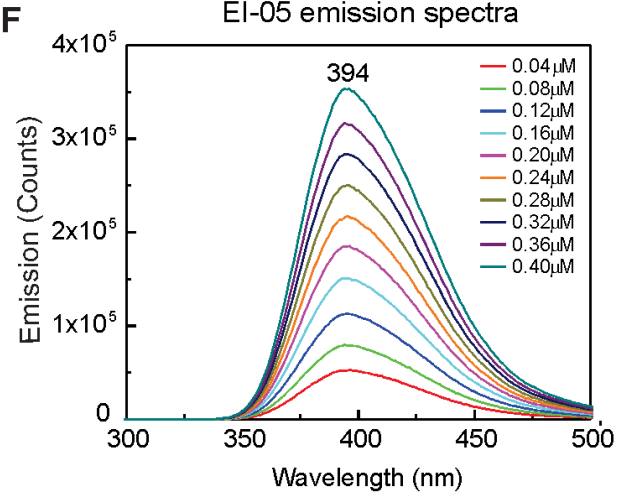

B

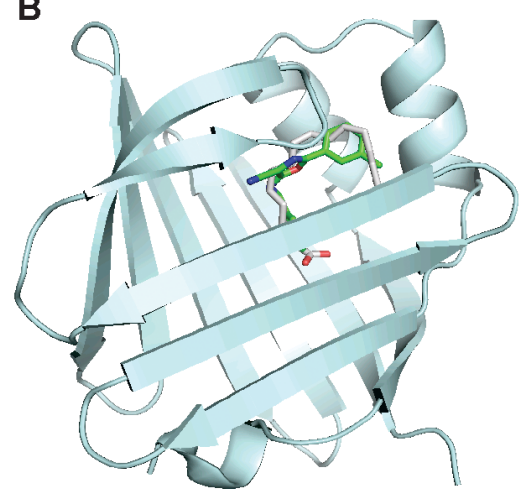

D El-05 emission and excition spectra
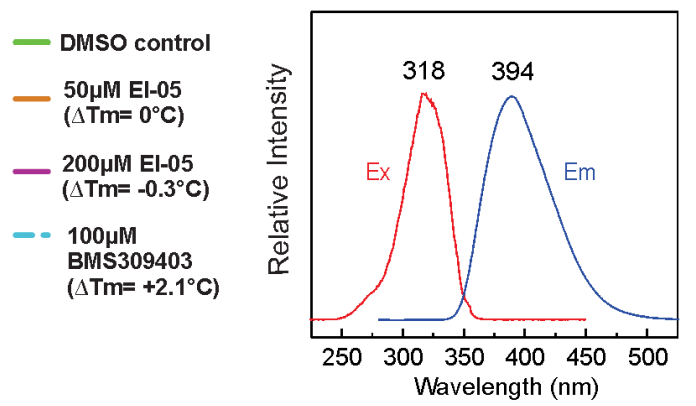

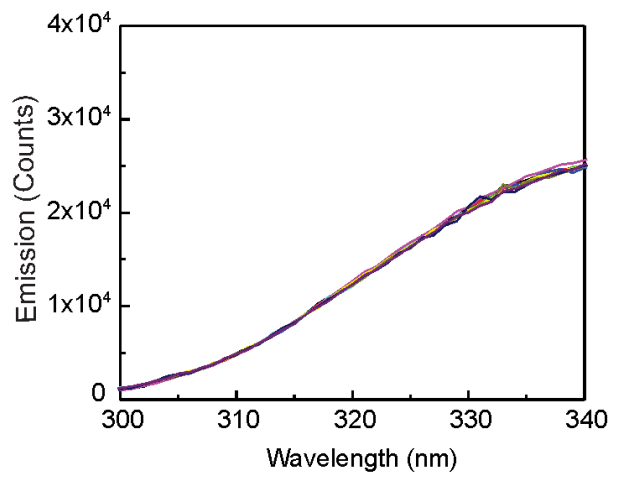

G

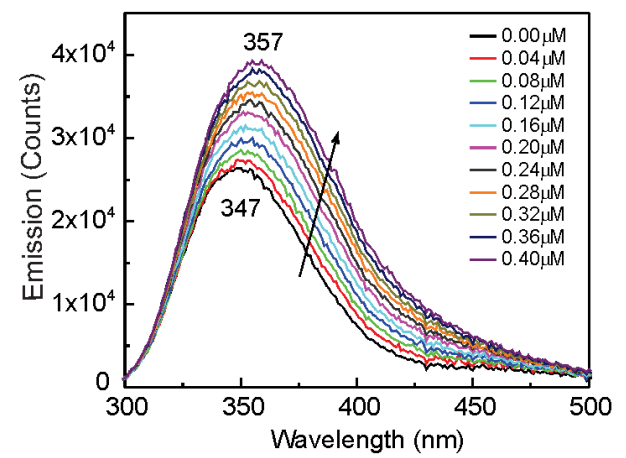

Figure 1: In-silico screening of EI-05. (A) Chemical structure of EI-05 (ZINC00467342) (B) The predicted model of EI-05 binding to the lipid-binding pocket of E-FABP. (C) Normalized melting curves depicting enhanced thermal stability of E-FABP by BMS309413 (blue dashed line), but not by EI-05 (orange and purple solid lines). (D) Excitation and emission spectra of EI-05 solved in methanol. (E) Tyr/Trp emission spectra of E-FABP $(0.5 \mu \mathrm{M})$ in the $300-500 \mathrm{~nm}$ range were measured by step-wise addition of indicated concentrations of EI-05. The Try/Trp emission signal between 300-347 nm (dashed box) is enlarged in the right panel. (F) The emission spectra of indicated concentrations of EI-05 in the absence of E-FABP. (G) Tyr/Trp emission of E-FABP $(0.5 \mu \mathrm{M})$ in the $300-500 \mathrm{~nm}$ range was measured by addition of indicated concentrations of BMS309413. Excitation at $270 \mathrm{~nm}$ was used for experiments shown in panels E, F and G. 
IFN $\beta$ production were dramatically inhibited by Tracsin $C$, a specific LD inhibitor (Figure 3G), further indicating the importance of LDs in mediating the production of IFN $\beta$ in macrophages. Of note, EI-05 treatment did not affect the expression of other FABP members, such as L-FABP and A-FABP, and the production of other tumor-related cytokines, such as TNF- $\alpha$, IL-6, IL-10, IL-12, iNOS, etc (Figure 4A-4H). When we further analyzed the production of IFN $\beta$ and other cytokines by peritoneal macrophages (PEMs), we confirmed that that EI-05 also enhanced IFN $\beta$ production in these physiologic populations (Figure 4I, 4J). These results indicate that EI-05 treatment greatly promotes E-FABP-mediated IFN $\beta$ production in macrophages.

\section{EI-05 treatment enhances macrophage activation}

Considering the critical role of IFN $\beta$ in activating antigen presenting cells $[18,19]$, we next measured the expression of MHCII and CD86 on macrophages in the presence or absence of EI-05 in vitro. As expected, EI-05 treatment not only increased the percentage of $\mathrm{MHCII}^{+}$ cells in $\mathrm{F} 4 / 80^{+} \mathrm{PEMs}$, but also upregulated the expression intensity of MHCII molecules (Figure 5A, 5B). CD86 expression was also significantly enhanced in response to EI-05 treatment (Figure 5C). To evaluate the relevance of these in vitro observations, we intraperitoneally injected mice with either EI-05 or the vehicle and analyzed the phenotypes of macrophages in vivo. Consistently, EI-05
A

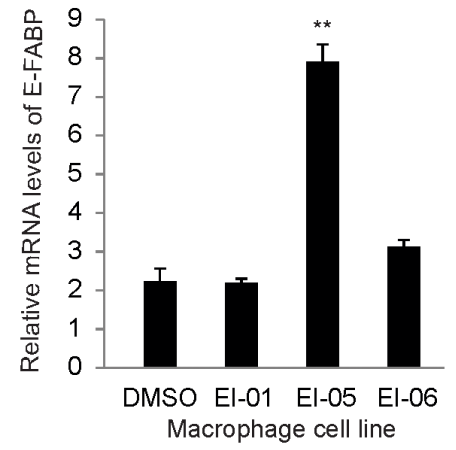

C

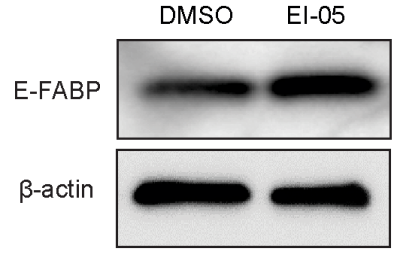

B
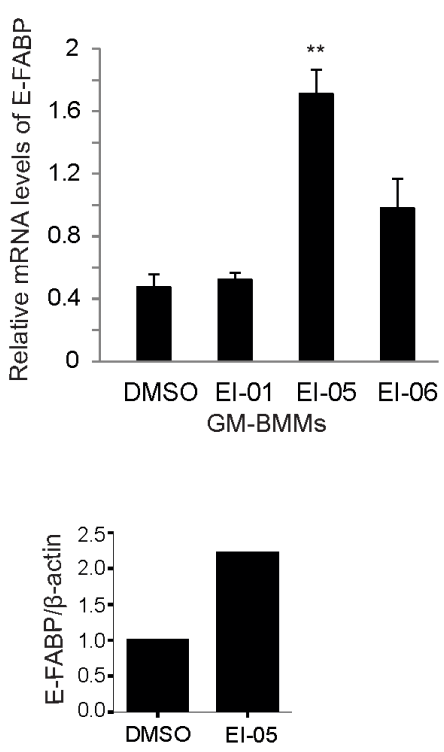
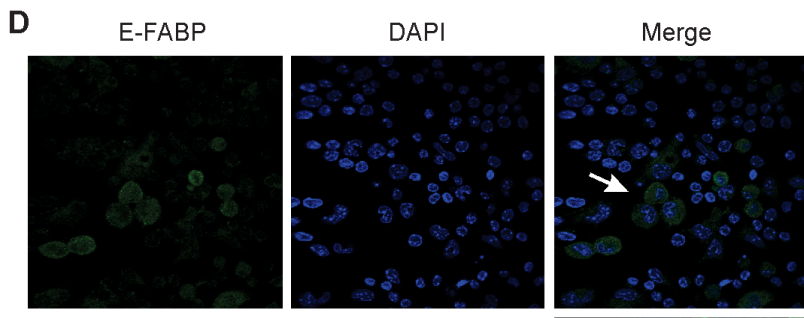

DMSO
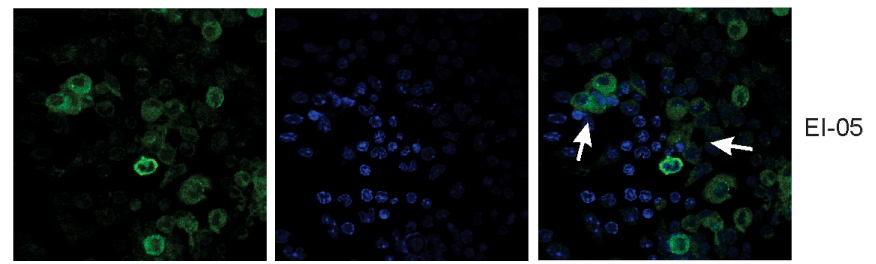

Figure 2: EI-05 enhances E-FABP expression in macrophages. Macrophages from a cell line (A) or bone-marrow (GM-BMMs) (B) were activated by LPS $(10 \mathrm{ng} / \mathrm{ml})$ in the presence of absence of screened E-FABP partners $(20 \mu \mathrm{M})$ for $24 \mathrm{~h}$ in vitro, respectively $(* *$, $\mathrm{p}<0.01$ as compared to DMSO group). E-FABP expression was quantified by qPCR. Mice were i.p. injected with EI-05 (10 mg/kg) and vehicle control for $24 \mathrm{~h}$, respectively. PBMCs were measured for E-FABP expression by western blot (C). Peritoneal macrophages were analyzed for E-FABP expression by confocal staining (D). 
A

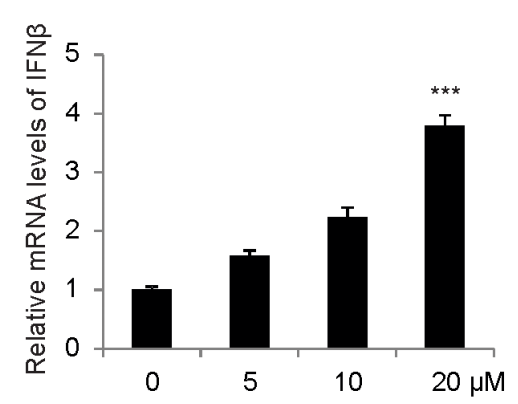

B

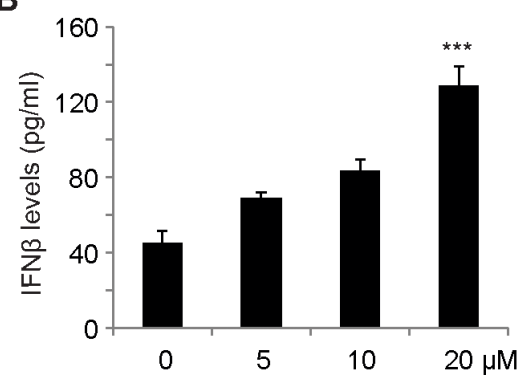

C

E5 ( $\mu \mathrm{M})$

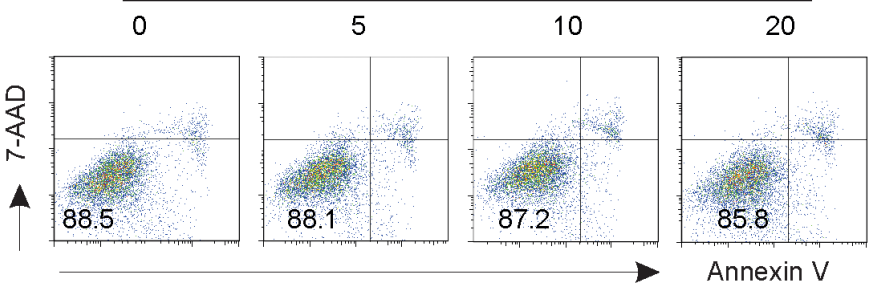

D

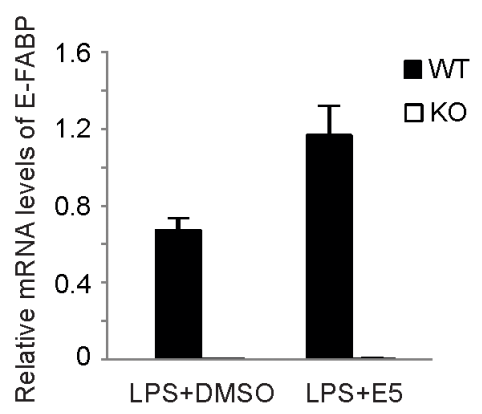

E

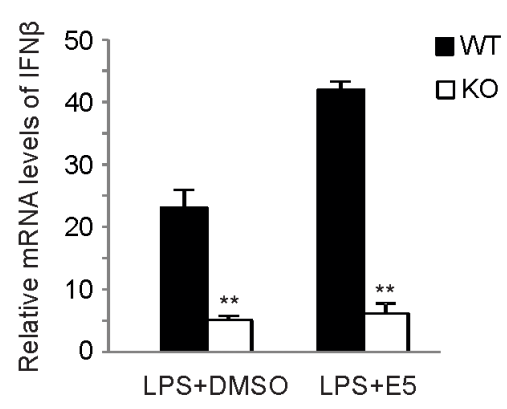

F
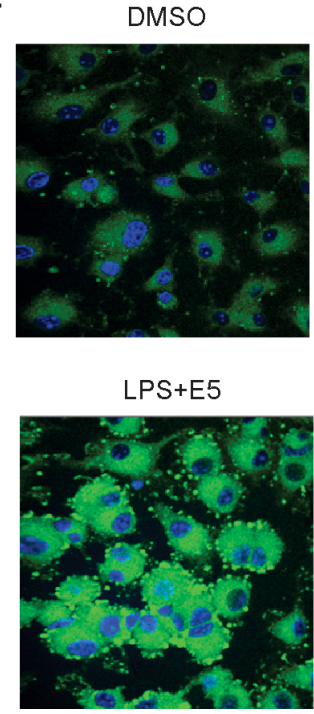

LPS+DMSO

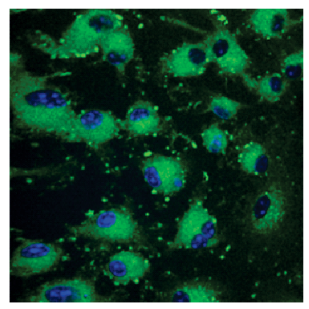

LPS+E5+Triacsin C

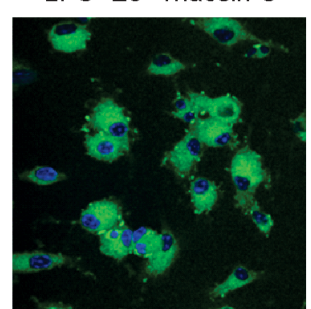

G

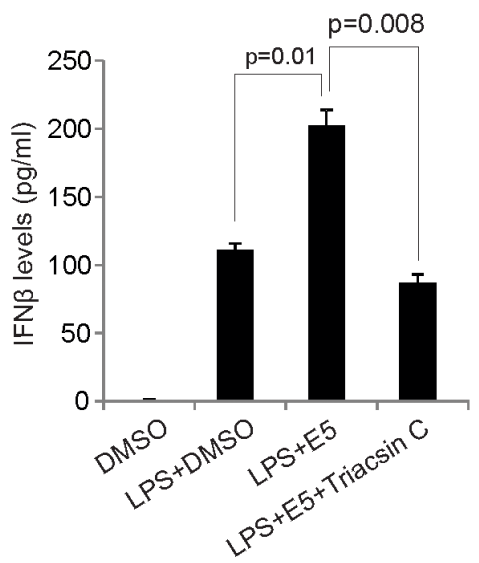

Figure 3: EI-05 treatment promotes IFN $\beta$ production in macrophages. GM-BMMs were activated by LPS (10 $\mathrm{ng} / \mathrm{ml})$ in the presence or absence of indicated concentrations of EI- 05 for $24 \mathrm{~h}$. IFN $\beta$ expression in macrophages was quantified by qPCR (A). IFN $\beta$ protein levels in cultural supernatants were measured by ELISA (B) (***, $<<0.001$ as compared to the control group). (C) Flow cytometric analysis of 7-AAD and annexin V expression on GM-BMMs treated with indicated concentrations of EI-05 for $24 \mathrm{~h}$. (D-E) E-FABP WT and KO macrophage cell lines were treated activated by LPS $(10 \mathrm{ng} / \mathrm{ml})$ in the presence of EI-05 or DMSO control for $3 \mathrm{~h}$. Expression of E-FABP (D) and IFN $\beta$ (E) was analyzed by realtime PCR (**, p $<0.01$ as compared to WT macrophages). (F) Confocal microscopy analysis of lipid droplet formation (BODIPY) in BM-GMMs with designated treatment with LPS (10 ng/ml), EI-05 (20 $\mu \mathrm{M})$ or Triacsin C (5 $\mu \mathrm{M})$. (G) Measurement of IFN $\beta$ levels in cultural supernatants of GM-BMMs with indicated treatment. 
A

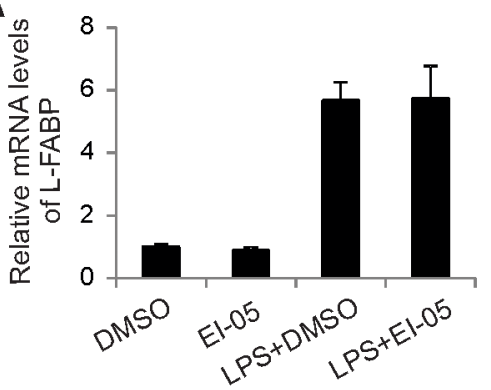

C

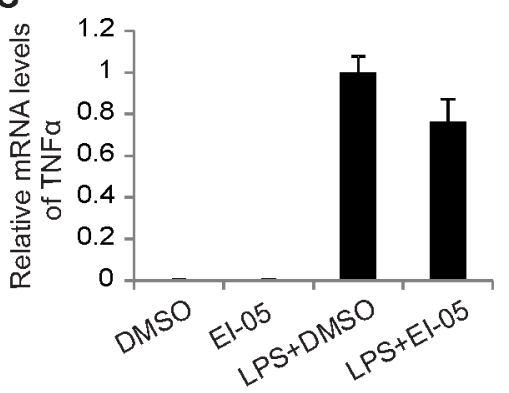

E

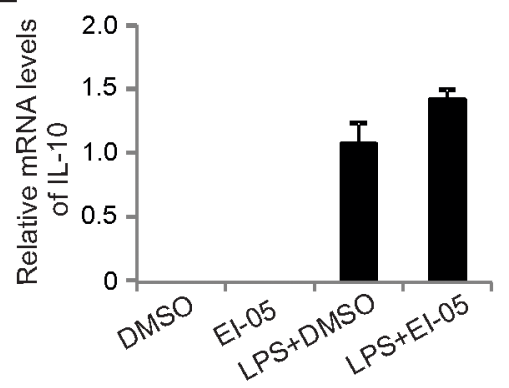

G

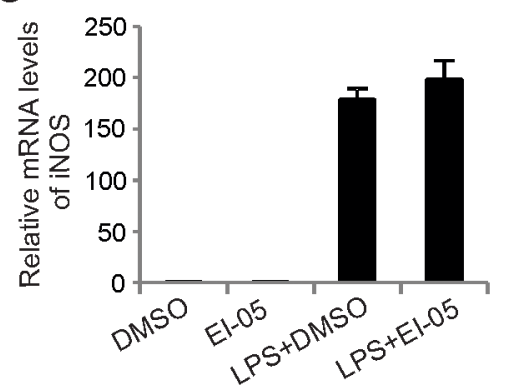

I

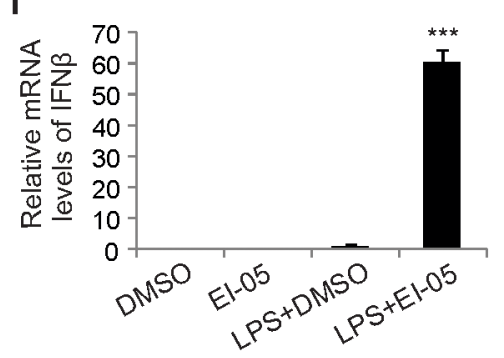

B

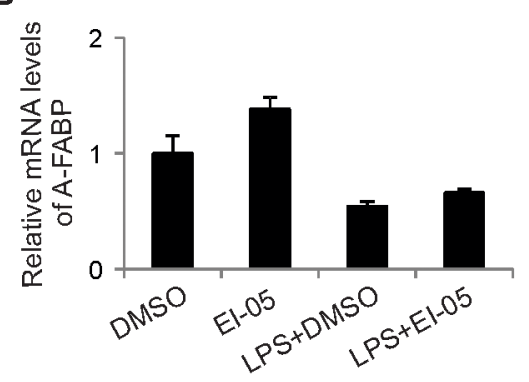

D

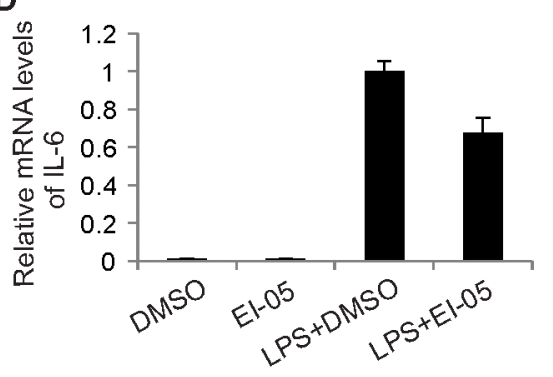

F

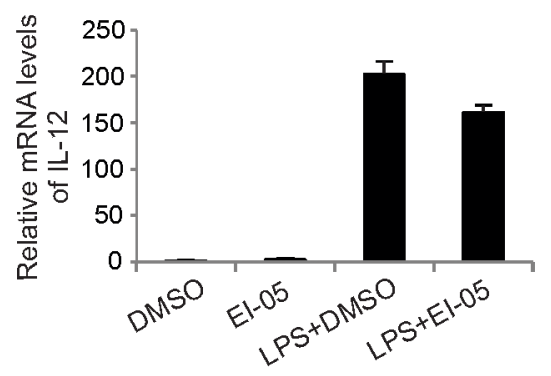

H

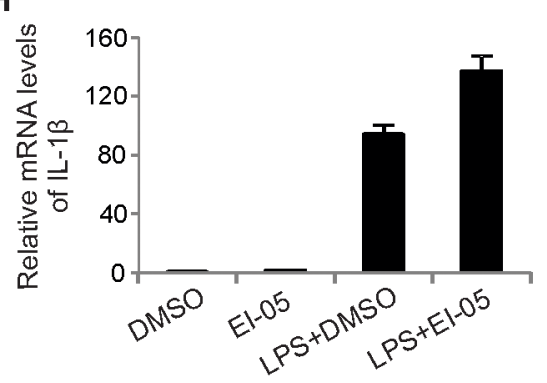

J

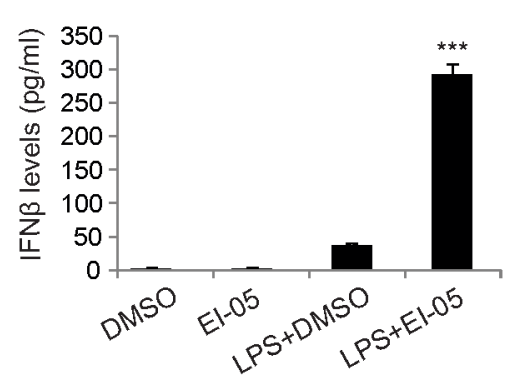

Figure 4: EI-05 treatment has no major impact on other tumor-related molecules in macrophages. (A-H) GM-BMMs were cultured with indicated treatment (EI-05, $20 \mu \mathrm{M}$; LPS $10 \mathrm{ng} / \mathrm{ml}$ ) for $24 \mathrm{~h}$. The expression of L-FABP, A-FABP and other tumor-related cytokines were quantified by qPCR. PEMs were cultured with indicated treatment (EI-05, $20 \mu \mathrm{M}$; LPS $10 \mathrm{ng} / \mathrm{ml}$ ) for $24 \mathrm{~h}$. Cells were lyzed for mRNA extraction and PCR analysis of relative RNA levels IFN $\beta$ (I). The protein levels of IFN $\beta$ in the cultural supernatants were measured by ELISA $(\mathrm{J})(* * *, \mathrm{p}<0.001$ as compared to the LPS+DMSO group). 

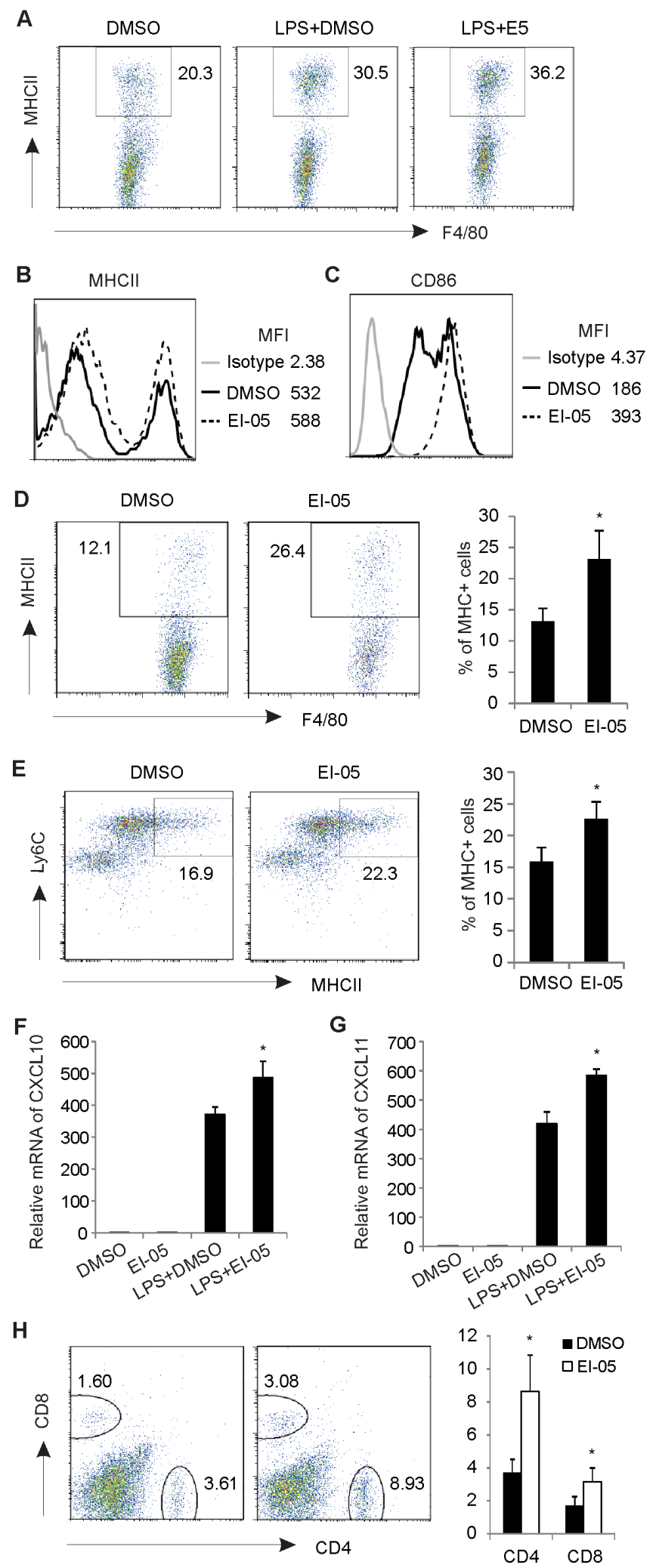

Figure 5: EI-05 promotes macrophage activation and $\mathrm{T}$ cell responses. Macrophages from peritoneum were collected and stimulated with LPS $(10 \mathrm{ng} / \mathrm{ml})$ in the presence or absence of EI-05 $(20 \mu \mathrm{M})$ for $24 \mathrm{~h}$ in vitro. (A) Flow cytometric analysis of MHCII populations in $\mathrm{F} 4 / 80^{+}$macrophages. Mean fluorescent intensity (MFI) of MHCII (B) and CD86 (C) expression on macrophages were analyzed by flow cytometry. Mice were i.p. injected with EI-05 or DMSO, respectively, for $24 \mathrm{~h}$. Flow cytometric analysis of $\mathrm{MHCII}^{+}$

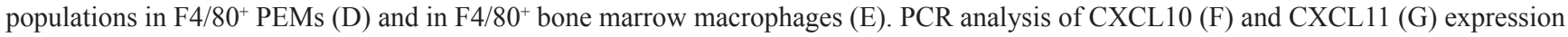
on macrophages activated by LPS or LPS plus EI- 05 for $24 \mathrm{~h}$. (H) Flow cytometric analysis of CD4 $4^{+}$and $\mathrm{CD} 8^{+} \mathrm{T}$ cell infiltration in peritoneum of mice administered i.p with either EI-05 or DMSO control ( ${ }^{*}, \mathrm{p}<0.05$ as compared to the DMSO group). 
administration significantly increased $\mathrm{MHCII}^{+}$populations in $\mathrm{F} 4 / 80^{+} \mathrm{PEMs}$ as compared to vehicle-treated mice (Figure 5D). In addition, mice treated with EI-05 exhibited elevated $\mathrm{MHCII}^{+}$macrophages in their bone marrow, suggesting a systemic effect of EI-05 (Figure 5E). Moreover, we measured IFN-stimulated chemokines in PEMs and demonstrated that EI-05 treatment greatly enhanced the expression of CXCL10 and CXCL11 (Figure 5F, 5G). Accordingly, there were increased infiltration of $\mathrm{CD}^{+} \mathrm{T}$ cells and $\mathrm{CD}^{+} \mathrm{T}$ cells in the peritoneum of EI05-treated mice in comparison to control mice (Figure $5 \mathrm{H})$. Altogether, our data suggest that EI-05 treatment greatly activates macrophages for antigen presentation, and subsequent $\mathrm{T}$ cell responses.

\section{EI-05 treatment reduces mammary tumor growth in vivo}

To investigate the therapeutic efficacy of EI-05 for tumor treatment, we orthotopically inoculated mouse E0771 mammary tumor cells into the mammary fat pat of mice and measured the tumor growth in EI-05- or vehicle-treated mice. Very strikingly, tumor growth in EI-05-treated mice was significantly decreased in volume (about 40\%) as compared to vehicle-treated group (Figure 6A). Accordingly, tumors in EI-05 treated mice exhibited reduced size and weight than those in control mice (Figure 6B, 6C). Interestingly, EI-05 treatment greatly increased the percentage of $\mathrm{F} 4 / 80^{+} \mathrm{CD} 11 \mathrm{c}^{+}$TAMs in tumors (Figure 6D). It is worth noting that EI-05 administration also remarkably enhanced the production of IFN $\gamma$ and Granzyme B by tumor-infiltrated $\mathrm{CD}^{+} \mathrm{T}$ cells (Figure 6E, 6F). Thus, these results suggest that EI-05 administration is able to inhibit mammary tumor growth, thus representing a novel drug candidate for anti-tumor treatment.

\section{DISCUSSION}

We have previously demonstrated that E-FABP expression in TAMs promotes their anti-tumor phenotype through enhanced IFN $\beta$ responses in mammary tumor models [8]. This study suggests E-FABP as a new protective factor that can enhance tumor immunosurveillance. Thus, identification of new chemicals or dietary factors which specifically upregulate E-FABP expression in macrophages may provide novel strategies for prevention and treatment of cancer. In this follow-up study, we identified EI-05 as a new E-FABP activator for inhibition of mammary tumor growth in a syngeneic mouse model.

To identify small molecules which may bind E-FABP and inhibit its activity and be used for treatment of autoimmune diseases, we screened more than one million compounds from the ZINC library using in-silico docking analysis with Glide XP $[15,20]$. Based on the score ranking and the molecular docking model against the E-FABP protein structure, EI-05 and several other compounds attracted our attention due to their potential binding to the lipid-binding pocket of E-FABP. However, when we conducted in vitro binding assays to detect protein-ligand interactions using various independent methods, such as fluorescent measurements, thermal shift assays and bead pull-down assays, none of the data showed any positive evidence of effective EI-05/E-FABP interactions. Because the positive binding of BMS309403 and other compounds to E-FABP were clearly observed in our assays, we were convinced that EI-05 actually does not bind to E-FABP (Figure 1). However, when we performed biological experiments with EI-05, we found that E-FABP is able to specifically enhance E-FABP expression in macrophages from different origins (Figure 2 ). Since the molecular mechanisms by which E-FABP is regulated remain largely unknown, it warrants further investigation to determine how EI-05 enhances E-FABP expression in macrophages.

Given that E-FABP upregulation in TAMs promotes IFN $\beta$ production and signaling to promote macrophage anti-tumor activity [8], we further analyzed whether EI-05 enhances IFN $\beta$ responses in macrophages. In fact, EI-05 treatment greatly enhances IFN $\beta$ production in both bone marrow-derived macrophages and PEMs (Figures 3, 4). In agreement with our previous studies, EI-05 markedly promotes LD formation in macrophages, which facilitates the enhanced production of IFN $\beta$. We noticed that LD inhibition does not completely inhibit EI-05-enhanced production of IFN $\beta$, thus, it is possible that EI-05 may also utilize other pathways to regulate IFN $\beta$ production. It has been shown that IFN $\beta$ production can be regulated by PPAR $\gamma$ in TLR3- and TLR4-stimulated macrophages [21]. We observed that EI-05 treatment promotes nuclear translocation of PPAR $\gamma$ and PPAR $\beta / \delta$ in macrophages (data not shown). Considering E-FABP as a target gene for PPAR $\beta / \delta$ [22], it is likely that EI-05 may regulate the transactivation of PPARs, thereby contributing to IFN $\beta$ production in macrophages.

Due to the critical role of type I IFNs in regulating innate and adaptive immunity, IFN $\alpha / \beta$ have been used in treatment of different types of tumors [23-25]. Unlike IFN $\gamma$ which acts directly on tumor cells, IFN $\alpha / \beta$ exerts its anti-tumor activity mainly through acting on immune populations, such as CD11 $\mathrm{c}^{+} \mathrm{APCs}, \mathrm{CD}^{+} \mathrm{T}$ cells, etc $[19$, 26]. For example, type I IFN endogenously produced by innate cells can bridge adaptive immune responses by upregulating MHC molecule expression on APCs, and enhancing Granzyme B-mediated tumor cytotoxicity of tumor infiltrated $\mathrm{CD}^{+} \mathrm{T}$ cells, all of which are supported by our data (Figures 5,6 ). However, it should be pointed out that exogenously administered $\operatorname{IFN} \alpha / \beta$ is often accompanied by many side effects, which extensively limit its clinical use. In addition, the pleiotropism and 

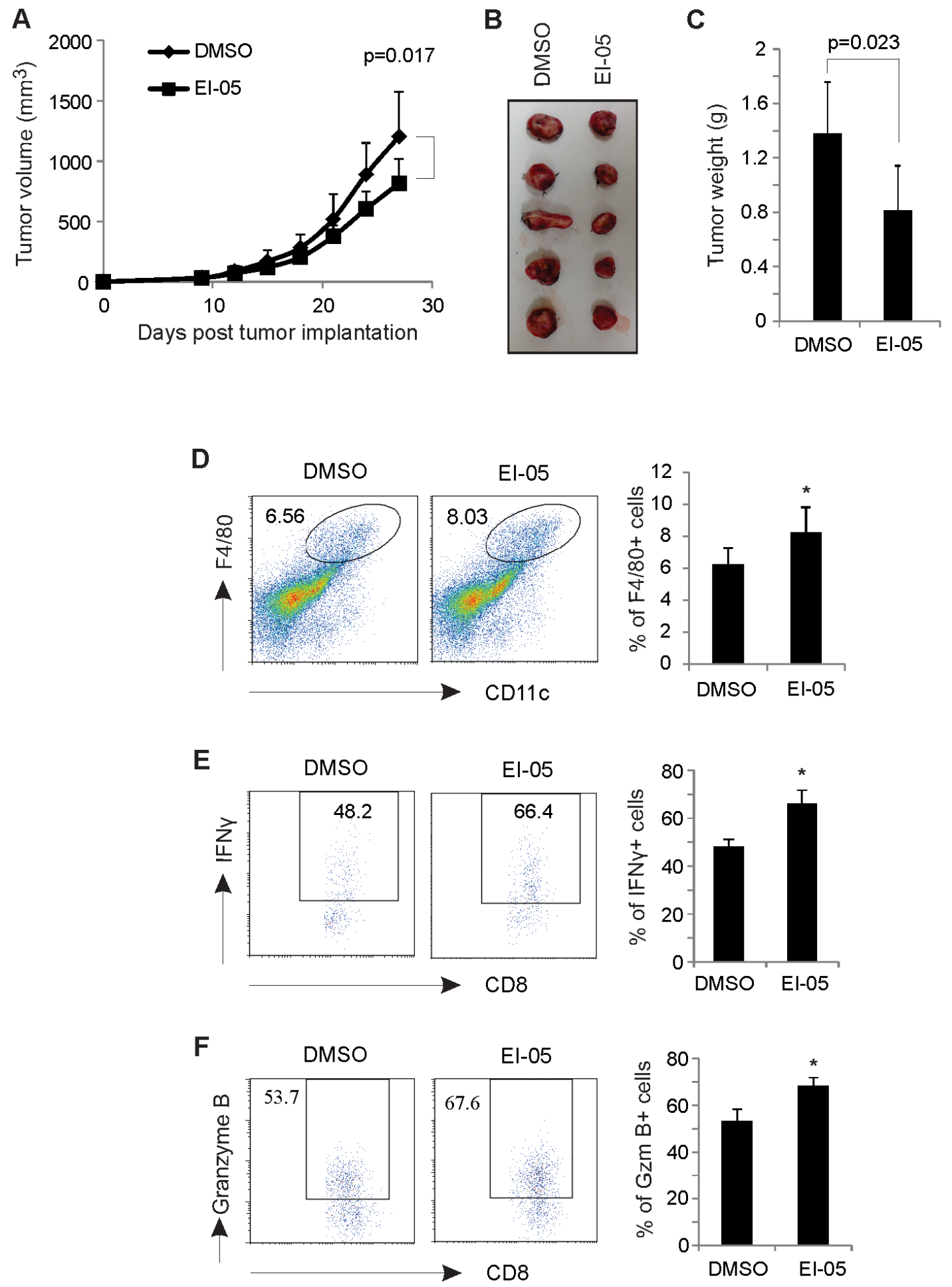

Figure 6: EI-05 treatment inhibits mammary tumor growth. E0771 tumor cells $\left(0.5 \times 10^{6} /\right.$ mouse $)$ were orthotopically injected into the mammary fat pad of C57B/C mice $(n=20)$. Mice were randomly divided into two groups $(n=10 /$ group $)$ and i.p. administered with either EI-05 (10 mg/kg) or vehicle DMSO control every other day. Tumors were measured at 3-day intervals and tumor growth curve were shown in panel (A). Representative tumor pictures in each group on day 27 after tumor injection were shown in panel (B). (C) Average tumor weights were measured on day 27 after mice were euthanized. (D) Flow cytometric analysis of tumor infiltrated $\mathrm{F} 4 / 80^{+} \mathrm{CD} 11 \mathrm{c}^{+}$ macrophages in EI-05- or DMSO-treated mice. Intracellular staining of the production of IFN $\gamma(\mathrm{E})$ and Granzyme B (F) in tumor infiltrated $\mathrm{CD}^{+} \mathrm{T}$ cells from EI-05- or DMSO-treated mice $(*, \mathrm{p}<0.05$ as compared to the DMSO group). 
redundancy of cytokine signaling make their use for immunotherapy a great challenge in vivo. It has been reported that exposure to exogenous IFN $\beta$ can decrease the expression of IFNGR1 and inhibit IFN $\gamma$-induced activation of macrophages [27]. Thus, the efficacy of direct application of exogenous IFN $\beta$ is very mixed and can be compromised due to the cross-regulation between type I and type II IFNs.

Since EI-05 can enhance E-FABP expression and endogenous IFN $\beta$ production by macrophages, it is logical to speculate that administering of EI-05 may be beneficial for anti-tumor therapy. To prove our concept, we first tested the toxicity of EI-05 application in vivo at the dose of $5 \mathrm{mg} / \mathrm{kg} / \mathrm{mice}, 10 \mathrm{mg} / \mathrm{kg} / \mathrm{mice}$ and $20 \mathrm{mg} / \mathrm{kg} /$ mice, respectively, based on other small molecules used in vivo [14]. All tested mice showed no toxic responses to EI-05 treatment regarding mouse weight, numbers of PBMCs, immune cell phenotype and cytokine production (data not shown), so we tested the efficacy of EI-05 using the middle dose at $10 \mathrm{mg} / \mathrm{kg}$ for tumor treatment with a syngeneic mouse mammary tumor model. Indeed, we demonstrated that EI-05 has great therapeutic efficacy in suppressing mammary tumor growth. Although more studies are under investigation to optimize the routes of administration and to test its efficacy in other tumors, EI-05 represents a promising drug candidate for anticancer treatment in many aspects. 1) It is clear that cell metabolism profoundly impacts their function [28, 29]. As the predominant FABP member in immune cells, E-FABP expression is critical in coordinating metabolism and function in immune cells [30]. Thus, enhancing E-FABP expression by EI-05 will facilitate their antitumor activity. 2) Due to the dual roles of IFN $\alpha / \beta$ in antitumor effect and pro-autoimmune responses [23], it is very hard to control the right dosage of IFN $\beta$ when it is administered exogenously. Instead, administering of EI05 will potentially reduce the side effects of exogenous IFN $\beta$ in that EI-05-enhanced IFN $\beta$ will be coordinately produced by host immune cells. 3) EI-05 itself shows a minimal toxic effect on either cultured cells or on mice when administered in vivo. Therefore, EI-05 has great potential clinical application.

In summary, we have identified EI-05 as a new activator for E-FABP. It not only enhances E-FABP expression in macrophages but also promotes their IFN $\beta$ production and responses. More importantly, administering EI-05 in vivo significantly suppresses mammary tumor growth. Thus, enhancing E-FABP activity by EI-05 may provide a new strategy for cancer prevention and treatment.

\section{METHODS}

\section{In silico screening for E-FABP modifiers}

Based on the crystal structure of E-FABP (PDB ID 1B56), in-silico screening was conducted using the program Glide v5.7 [15]. Flexible docking was performed with the standard precision (SP) mode. More than one million compounds from the ZINC database were screened for potential E-FABP modifiers. The top-ranked compounds were clustered into different classes based on similarity of chemical structures. Compound EI-05 was selected due to its potential interaction with lipid binding pocket of E-FABP. EI-05 was purchased from Interbio Screen Ltd for biological experimental tests.

\section{In vitro binding assays}

Recombinant E-FABP was expressed in BL21 (DE3) and purified using Ni-NTA resin followed by the cleavage of His-tag and ion-exchange chromatography. Endogenous lipids bound to E-FABP were removed by running through the Lipidex-1000 column. Binding of EI05/E-FABP was determined by two independent assays, in addition to a compound-coupled bead pull-down assay. For the thermal shift assay [31], reactions were set-up in PCR tubes in a $20 \mu \mathrm{l}$ volume containing $10 \mu \mathrm{M}$ E-FABP and $10 \times$ SYPRO Orange dye (Invitrogen) in $20 \mathrm{mM}$ HEPES pH 7 and $150 \mathrm{mM} \mathrm{NaCl}$, containing either EI05 , BMS or DMSO control. PCR tubes were then sealed, centrifuged and heated from $25^{\circ} \mathrm{C}$ to $95^{\circ} \mathrm{C}$ at a rate of 1 degree/min on 7500 Real-Time PCR machine (Applied Biosystems). For the fluorescence assay, we measured the fluorescent signals of protein Trp as previously described [32]. Briefly, emission spectra of E-FABP Tyr/Trp and EI05 were measured using Fluorolog-3 Spectrofluorometer (Horiba Scientific). Emission spectra were recorded from 300 to $500 \mathrm{~nm}$ in the presence and absence of E-FABP $(0.5 \mu \mathrm{M})$ with addition of indicated concentrations of EI05 or BMS-309413 (positive control) at $25^{\circ} \mathrm{C}$.

\section{Generation of macrophages from bone marrow or peritoneum}

For generation of GM-CSF-induced bone marrowderived macrophages (GM-BMMs), bone marrow from mouse femurs or tibias was flushed with PBS, supplemented with 2\% FBS. After lysis of red blood cells (R\&D systems), the cells were plated in 100-mm tissue culture dishes with 5\% FBS RPMI 1640 medium at $37^{\circ} \mathrm{C} / 5 \% \mathrm{CO}_{2}$ for 4 hours. Then the non-adherent cells were plated in 5\% FBS RPMI 1640 medium with $20 \mathrm{ng} /$ ml GM-CSF (R\&D Systems). New 5\% FBS RPMI 1640 
medium with $20 \mathrm{ng} / \mathrm{ml}$ GM-CSF was added on day 2 and day 5. The cultured GM-BMMs were collected on day 7 for further experiments. For collecting peritoneal macrophages (PEMs), cells from peritoneum were flushed out with $10 \mathrm{ml}$ PBS and plated in 24-well plate for 2 hours. After wash off the non-attached cells, attached PEMs were used for experiments.

\section{Real-time PCR}

For real-time PCR analyses, RNA was extracted from primary macrophages or macrophage cell lines using RNeasy Mini Kit (Qiagen). cDNA synthesis was performed with QuantiTect Reverse Transcription Kit (Qiagen). Quantitative PCR was performed with SYBR ${ }^{\circledR}$ Green PCR Master Mix using ABI 7500 Real-Time PCR Systems (Applied Biosystems). E-FABP, IFN $\beta$, CXCL10, CXCL11, PPAR $\gamma$, TNF $\alpha$, IL-6, IL-10 and $\beta$-actin expression was analyzed by QuantiTect primer assays (Qiagen). Results were normalized to $\beta$-actin. Relative expression of the target genes was measured using the $\Delta \Delta \mathrm{CT}$ approach.

\section{Confocal analysis}

Macrophages from peripheral blood or peritoneum cultured on poly-D-lysine coated coverslips (NeuVitro) in a 24-well plate were treated with LPS $(10 \mathrm{ng} / \mathrm{ml})$ in the presence or absence of EI-05 $(20 \mu \mathrm{M})$ for $24 \mathrm{~h}$. After fixation and permeabilization, the cells were stained with E-FABP specific antibody (R\&D System). Confocal analysis was performed with Nikon Eclipse TE2000 confocal microscopy.

\section{ELISA}

GM-BMMs or PEMs were stimulated with LPS (10 $\mathrm{ng} / \mathrm{ml}$ ) in the presence or absence of EI-05 for 24 hours. Culture supernatants were collected for measurement of protein levels of IFN $\beta$ with mouse ELISA kits (Biolegend) according to manufacturer's instructions.

\section{Western blot}

To quantify the protein levels of E-FABP in macrophages, macrophages from peripheral blood or peritoneum with designated treatments were lysed in $1 \times$ lysis buffer with protease and phosphorylation inhibitors. Protein concentration was determined by BCA assay (Thermo Scientific). $10 \mu \mathrm{g}$ of total protein was loaded. $\beta$-actin was quantified as a loading control. Mouse E-FABP antibody (goat) was from R\&D Systems. Mouse $\beta$-actin antibodies (rabbit) were from Cell Signaling Technology.

\section{Flow cytometric analysis}

Immune cells from peritoneum or tumors were subjected to surface staining or cultured with PMA (5 ng/ $\mathrm{ml}$; Sigma), ionomycin (500 ng/ml; Sigma) and Golgiplug (BD) for $6 \mathrm{hrs}$, harvested for surface and intracellular staining. Flow cytometric data were collected with a BD FACS CaliburTM. Flow cytometric data analyses were performed with Flowjo (Tree Star). The following antibodies were used for cell staining: anti-CD4 (clone RM4-5), anti-CD8 (clone 53-6.7), anti-MHC class II (clone M5/114.15.2), anti-IFN $\gamma$ (clone XMG1.2), antiGranzyme B (clone GB11), anti-CD80 (clone 16-10A1) and anti-CD86 (clone GL-1), anti-CD11c (clone HL3) and anti-F4/80 (clone BM8).

\section{Mice and tumor model}

C57BL/6 mice were bred and maintained in the animal facility at the Hormel Institute in accordance with the University of Minnesota Institutional Animal Care and Use Committee (IACUC). Six to ten-weekold female mice were fed standard chow diet and utilized for these experiments. All animal protocols were approved by IACUC in the University of Minnesota and followed national guidelines. E0771 cell line derived from a C57BL/6 mouse mammary adenocarcinoma was orthotopically injected into the mammary fat pad of the mice. EI-05 (10 mg/kg) or vehicle control were i.p. injected every other day until mice were sacrificed on day 27 after tumor cell injection. Tumors were measured at 3 day intervals with calipers at two bisecting diameters and an approximate volume was calculated by the formula $(0.4) \times($ large diameter $) \times(\text { small diameter })^{2}$ and single cells from tumor were prepared as previously described for analysis $[8,33]$.

\section{Statistical analysis}

All quantitative data were shown as means \pm SD. Unpaired, two-tailed Student's t-test was performed for comparison of results from different treatments. P value less than 0.05 is considered statistically significant.

\section{ACKNOWLEGEMENTS}

This study is supported in part by the NIH R01 grants (CA177679, CA180986, CA157012, GM45928), and Career Transition Fellowship (TA3047-1) from the National Multiple Sclerosis Society.

\section{CONFLICTS OF INTEREST}

The authors declare no conflict of interest. 


\section{REFERENCES}

1 Smyth MJ, Dunn GP, Schreiber RD. Cancer immunosurveillance and immunoediting: the roles of immunity in suppressing tumor development and shaping tumor immunogenicity. Adv Immunol 2006;90:1-50.

2 Swann JB, Smyth MJ. Immune surveillance of tumors. J Clin Invest 2007;117:1137-46.

3 Pearce EL, Pearce EJ. Metabolic pathways in immune cell activation and quiescence. Immunity 2013;38:633-43.

4 Furuhashi M, Hotamisligil GS. Fatty acid-binding proteins: role in metabolic diseases and potential as drug targets. Nat Rev Drug Discov 2008;7:489-503.

5 Hertzel AV, Bernlohr DA. The mammalian fatty acidbinding protein multigene family: molecular and genetic insights into function. Trends Endocrinol Metab 2000;11:175-80.

6 Storch J, Corsico B. The emerging functions and mechanisms of mammalian fatty acid-binding proteins. Annu Rev Nutr 2008;28:73-95.

7 Zhang Y, Li B. E-FABP: regulator of immune function. Oncoscience 2014;1:398-9.

8 Zhang Y, Sun Y, Rao E, Yan F, Li Q, Zhang Y, et al. Fatty acid-binding protein E-FABP restricts tumor growth by promoting IFN-beta responses in tumor-associated macrophages. Cancer Res 2014;74:2986-98.

9 Li B, Reynolds JM, Stout RD, Bernlohr DA, Suttles J. Regulation of Th17 differentiation by epidermal fatty acidbinding protein. J Immunol 2009; 182:7625-33.

10 Hertzel AV, Hellberg K, Reynolds JM, Kruse AC, Juhlmann $\mathrm{BE}$, Smith AJ, et al. Identification and characterization of a small molecule inhibitor of Fatty Acid binding proteins. J Med Chem 2009;52:6024-31.

11 Lehmann F, Haile S, Axen E, Medina C, Uppenberg J, Svensson S, et al. Discovery of inhibitors of human adipocyte fatty acid-binding protein, a potential type 2 diabetes target. Bioorg Med Chem Lett 2004;14:4445-8.

12 Liu X, Huang X, Lin W, Wang D, Diao Y, Li H, et al. New aromatic substituted pyrazoles as selective inhibitors of human adipocyte fatty acid-binding protein. Bioorg Med Chem Lett 2011;21:2949-52.

13 Sulsky R, Magnin DR, Huang Y, Simpkins L, Taunk P, Patel M, et al. Potent and selective biphenyl azole inhibitors of adipocyte fatty acid binding protein (aFABP). Bioorg Med Chem Lett 2007;17:3511-5.

14 Furuhashi M, Tuncman G, Gorgun CZ, Makowski L, Atsumi G, Vaillancourt E, et al. Treatment of diabetes and atherosclerosis by inhibiting fatty-acid-binding protein aP2. Nature 2007;447:959-65.

15 Friesner RA, Banks JL, Murphy RB, Halgren TA, Klicic JJ, Mainz DT, et al. Glide: a new approach for rapid, accurate docking and scoring. 1. Method and assessment of docking accuracy. J Med Chem 2004;47:1739-49.

16 Sanson B, Wang T, Sun J, Wang L, Kaczocha M, Ojima I, et al. Crystallographic study of FABP5 as an intracellular endocannabinoid transporter. Acta Crystallogr D Biol Crystallogr 2014;70:290-8.

17 O'Neill LA. Immunology. Sensing the dark side of DNA. Science 2013;339:763-4.

18 Simmons DP, Wearsch PA, Canaday DH, Meyerson HJ, Liu YC, Wang Y, et al. Type I IFN drives a distinctive dendritic cell maturation phenotype that allows continued class II MHC synthesis and antigen processing. J Immunol 2012;188:3116-26.

19 Smyth MJ. Type I interferon and cancer immunoediting. Nat Immunol 2005;6:646-8.

20 Xie H, Lee MH, Zhu F, Reddy K, Huang Z, Kim DJ, et al. Discovery of the novel mTOR inhibitor and its antitumor activities in vitro and in vivo. Mol Cancer Ther 2013;12:950-8.

21 Zhao W, Wang L, Zhang M, Wang P, Zhang L, Yuan C, et al. Peroxisome proliferator-activated receptor gamma negatively regulates IFN-beta production in Toll-like receptor (TLR) 3- and TLR4-stimulated macrophages by preventing interferon regulatory factor 3 binding to the IFNbeta promoter. J Biol Chem 2011;286:5519-28.

22 Morgan E, Kannan-Thulasiraman P, Noy N. Involvement of Fatty Acid Binding Protein 5 and PPARbeta/delta in Prostate Cancer Cell Growth. PPAR Res 2010;2010.

23 Trinchieri G. Type I interferon: friend or foe? J Exp Med 2010;207:2053-63.

24 Moschos S, Kirkwood JM. Present role and future potential of type I interferons in adjuvant therapy of highrisk operable melanoma. Cytokine Growth Factor Rev 2007;18:451-8.

25 Gresser I, Belardelli F. Endogenous type I interferons as a defense against tumors. Cytokine Growth Factor Rev 2002;13:111-8.

26 Diamond MS, Kinder M, Matsushita H, Mashayekhi M, Dunn GP, Archambault JM, et al. Type I interferon is selectively required by dendritic cells for immune rejection of tumors. J Exp Med 2011;208:1989-2003.

27 Trinchieri G. Type I interferon: friend or foe? J Exp Med 2010;207:2053-63.

28 Ghesquiere B, Wong BW, Kuchnio A, Carmeliet P. Metabolism of stromal and immune cells in health and disease. Nature 2014;511:167-76.

29 Biswas SK, Mantovani A. Orchestration of metabolism by macrophages. Cell Metab 2012;15:432-7.

30 Rolph MS, Young TR, Shum BO, Gorgun CZ, SchmitzPeiffer C, Ramshaw IA, et al. Regulation of dendritic cell function and $\mathrm{T}$ cell priming by the fatty acid-binding protein AP2. J Immunol 2006;177:7794-801.

31 Niesen FH, Berglund H, Vedadi M. The use of differential scanning fluorimetry to detect ligand interactions that promote protein stability. Nat Protoc 2007;2:2212-21.

32 Zhai X, Malakhova ML, Pike HM, Benson LM, Bergen HR, III, Sugar IP, et al. Glycolipid acquisition by human 
glycolipid transfer protein dramatically alters intrinsic tryptophan fluorescence: insights into glycolipid binding affinity. J Biol Chem 2009;284:13620-8.

33 Watkins SK, Egilmez NK, Suttles J, Stout RD. IL-12 rapidly alters the functional profile of tumor-associated and tumor-infiltrating macrophages in vitro and in vivo. $\mathrm{J}$ Immunol 2007;178:1357-62. 